\title{
Wettbewerbsfähigkeit durch Nachhaltigkeitsorientierung
}

\author{
Welches Interesse haben Unternehmen an Nachhaltigkeitsstrategien? Was sind \\ die wesentlichen Anknüpfungspunkte für Kooperationen, um solche Strategien \\ zu entwickeln? Ausgehend von Erfahrungen aus dem Projekt Nachhaltige Me- \\ tallwirtschaft Hamburg (1) lassen sich wesentliche Motive und Triebkräfte be- \\ stimmen. Auf den zunehmend nachfragedominierten und dynamischen Märkten \\ dürfte sich eine Nachhaltigkeitsorientierung strategisch auszahlen.
}

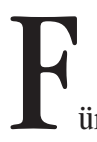

\section{Von Arnim von Gleich,}

Manuel Gottschick und Dirk Jepsen ür das Interesse von Unternehmen an einer nachhaltigeren Wirtschaftsweise sind mindestens drei Gruppen von Motiven denkbar:

1. Überzeugungen von Produzenten und Konsumenten (ethische Produktion und ethischer Konsum),

2. Realisierung von Kostenvorteilen (weniger Stoff- und Energieeinsatz, Ressourceneffizienz),

3. Langfristige Marktstrategien (Wettbewerbs- und Innovationsfähigkeit).

Auf allen drei Wegen lassen sich Unternehmen ansprechen, mit je spezifischen Vor- und Nachteilen.

\section{- Motiv Überzeugungen}

Das Anknüpfen an der ethischen Verantwortung der Unternehmen ist recht effektiv, weil das Thema von Anfang an meist „Chefsache“ ist. Die möglichen Kooperationspartner beschränken sich aber auf der Seite der Hersteller und Dienstleister auf immer wieder die gleichen Mitglieder der ökologischen Unternehmensverbände B.A.U.M. und future. Auch die Produktgruppen, in denen ethischer Konsum eine nennenswerte Rolle spielt, sind noch recht übersichtlich: Nahrungsmittel, Kosmetika, Papier, der Bausektor, zum Teil wohl auch Textilien. Über ethischen Konsum im Bereich der Metallwaren ist wenig bekannt.

\section{Motiv Ressourceneffizienz}

Der Versuch, mit Unternehmen zu kooperieren, die noch nicht von der Relevanz des Nachhaltigkeitszieles in der alltäglichen Unternehmenspraxis überzeugt sind, beginnt heute meist mit der Identifikation sogenannter Win-win-win-Strategien, wobei die Steigerung der Ressourceneffizienz an erster Stelle steht. Wenn es uns gelänge, mit weni- ger Stoff- und Energieeinsatz und damit weniger Kosten dasselbe Produkt oder dieselbe Dienstleistung herzustellen und dabei auch noch soziale Fortschritte zu realisieren, wäre das ein Gewinn für alle drei Dimensionen der Nachhaltigkeit.

Sehr schnell stellt sich dann aber heraus, dass ein Unternehmen auch in der Lage sein muss, solche Effizienzgewinne zu realisieren. Das ist alles andere als trivial. Die Win-win-win-Strategie Verbesserung der Ressourceneffizienz hat offenbar eine zweite Win-win-win-Strategie zur Voraussetzung: die Verbesserung der Innovationsfähigkeit. Die Hürden, die dafür zu überwinden sind, reichen vom ,not invented here“-Syndrom über Abteilungsegoismen und Kommunikationsblockaden bis zur berühmten Feststellung: „Machbar ist, was der Chef versteht".

Es macht auch einen großen Unterschied, welche Person oder Abteilung externe Akteure als Ansprechpartner im Unternehmen erreichen können. Beim Ansatz der Ressourceneffizienz ist es meist die Umweltabteilung, die darunter häufig nur Abfallmanagement versteht. Eine umfassende Nachhaltigkeitsstrategie ist in solchen Fachabteilungen oft schwer zu vermitteln. Die Umweltabteilung gehört auch nicht unbedingt zu den mächtigen Abteilungen im Unternehmen oder zu denen, mit deren Hilfe man glaubt, Marktanteile sichern oder gar erobern zu können. Ihre Arbeit wurde bisher eher als kostenerzeugend und als Innovationsbremse wahrgenommen. Initiativen aus dieser Richtung sind daher keine Selbstläufer.

Nehmen wir das Beispiel zweier 500.000 MarkProjekte, die als Vorschläge in den Unternehmensvorstand eingebracht werden: eines zur Verbesserung der Ressourceneffizienz und ein zweites für eine Marktoffensive inklusive Werbekampagne. Selbst wenn es bei beiden Projekten gleich ungewiss ist, ob sie sich am Ende für das Unternehmen wirklich rechnen, müssen wir davon ausgehen, dass die Marktoffensive größere Chancen hat. Das Ressourcenprojekt hingegen wird eher in die Abteilung zurückverwiesen, mit der Auflage, den erwartbaren Gewinn belastbarer zu kalkulieren und zu belegen.

Es käme also darauf an, die Nachhaltigkeitsorientierung in der strategischen Unternehmensplanung zu verankern, sie (zumindest auch) als ein Element zu verstehen, mit dessen Hilfe langfristig Marktpositionen gesichert und verbessert werden können. Das hätte zugleich auch zur Folge, dass Kooperationen mit stärkeren Abteilungen stattfinden, mit der Produktentwicklung, der Qualitätssicherung, dem Verkauf oder eben direkt mit der Unternehmensleitung, zu deren ureigenster Aufgabe die längerfristige Unternehmensstrategie ja gehört.

Auch wenn ökologische Fragen in die Praxis moderner Unternehmen längst integriert sind, ist die Verankerung der Nachhaltigkeitsorientierung in der strategischen Unternehmensplanung überfällig. Ressourceneffizienz und Stoffstrommanagement werden häufig auf Abfallvermeidung verkïrzt, gegebenenfalls noch erweitert um Probleme der Logistik oder des Vertriebs. Die in der Produktgestaltung und der Produktnutzung liegenden Möglichkeiten liegen oft noch brach. Nicht zufällig erlangte der Begriff der Produktverantwortung seine heutige Bedeutung erst durch staatliche Regulation, durch das Kreislaufwirtschafts- und Abfallgesetz.

Mit einem realistischen Blick auf die bundesrepublikanische Wirklichkeit wird man trotz alledem heute zu dem Schluss kommen, dass die Unternehmen derzeit die erfolgreichsten Akteure der ökologischen Modernisierung sind. Doch Nachhaltigkeit meint mehr als ökologische Modernisierung.

\section{Motiv Wettbewerbs- und Innovationsfähigkeit}

Veränderungen in der Produktentwicklung, Produktgestaltung und Produktnutzung sind allerdings um Größenordnungen schwieriger und komplexer als die Optimierung von Produktionsprozessen. Zudem erfordern sie die Öffnung des Unternehmens gerade in einem Bereich, der gerne dem Wettbewerber gegenüber geheim halten wird. Bei Produktstrategien sind die Unternehmen - je nach der Art der Märkte, in denen sie agieren (siehe unten) - extrem abhängig von der quantitativen und qualitativen Nachfragesituation. Und auf den 
Märkten vollzogen sich in den vergangenen Jahrzehnten wesentlich dramatischere Veränderungen als in der internen Unternehmenswirklichkeit, ja man kann sagen, dass letztere im Wesentlichen auf erstere reagieren. Mit dem Begriff Globalisierung wird dabei nur ein Aspekt umschrieben.

Der Wettbewerb verschärft sich aber nicht nur quantitativ, weil neue Wettbewerber auftreten, sondern vor allem auch qualitativ, weil sich die Märkte und die Nachfrage selbst verändern, sie werden fragmentierter, dynamischer und damit unübersichtlicher. Radikale Kundenorientierung und die Steigerung der Innovationsfähigkeit, der Fähigkeit von Unternehmen, sich flexibel auf derartige Veränderungen auf den Märkten einzustellen, ja sie zu antizipieren, gehören damit zu den wichtigsten Erfolgsfaktoren.

Wie lässt sich aber Wettbewerbsfähigkeit bzw. die vom Kunden gewünschte Produktqualität genau bestimmen? Auf jeden Fall handelt es sich um qualitative Aspekte. Mit in Mark und Pfennig ausdrückbaren Produktpreisen, aktuellen Umsatzzahlen oder Renditen ist das Phänomen nicht hinreichend zu erfassen. Man kann sehr wohl kurzfristig hohe Gewinne einfahren und dabei die langfristige Wettbewerbsfähigkeit ruinieren. Auch die Kunden interessiert nicht das billigste Produkt, sondern Preis und Leistung. Und zur Leistung bzw. Produktqualität kann sehr viel gehören, angefangen von Lieferfristen über Garantieleistungen und Service bis hin zur ökologischen und sozialen Performance des Unternehmens. Qualität ist, was der Kunde darunter versteht. Dass die Kunden das Unternehmen ,erziehen“ ist sehr viel wahrscheinlicher als der umgekehrte Fall, auch wenn das Unternehmen noch so viel Geld für Public relations ausgibt. Natürlich interessiert der Preis nach wie vor, insbesondere dann, wenn die Qualität vergleichbar ist. Was passiert aber, wenn - wie es heute oft der Fall ist - Preis und Qualität im engeren Sinne bei mehreren Wettbewerbern vergleichbar sind? Dann kann plötzlich jeder vom Kunden wahrnehmbare Zusatznutzen entscheidend für den Verkaufserfolg werden, angefangen vom optischen Design, über die Marke, bis hin zum Vorhanden- bzw. Nichtvorhandensein eines Umweltmanagementsystems nach ISO 14.001 oder EMAS. Das gilt nicht nur für business to consumer-, sondern auch für business to business-Beziebungen.

In dieser Situation können damit auch normative Aspekte an Bedeutung gewinnen, die bisher als weiche Faktoren eher belächelt wurden. Damit eröffnen sich neue Handlungsspielräume für
Nachhaltigkeitsstrategien. Ein gutes Image ist dann kein Luxus mehr, auf den man genauso gut verzichten könnte, sondern wesentlicher Teil der Wettbewerbsfähigkeit. Nicht zuletzt Unternehmen, die an die Börse gegangen sind, mussten die oft schmerzhafte Erfahrung machen, von wie viel qualitativen Aspekten der Kurs ihrer Aktien inzwischen beeinflusst wird. Gerade an der Börse wird deutlich, wie viel Psychologie heute die scheinbar so rationale ökonomie bestimmt, wie Erwartungen wichtiger werden als exakte schwarze Zahlen.

\section{- Von der Masse zur differenzierten Qualität}

Doch wie verallgemeinerbar sind solche Veränderungen bzw. strategischen Anknüpfungspunkte? Für welche Unternehmen, Märkte, Produktions- und Produktstrategien treffen sie mehr und für welche weniger $\mathrm{zu}$ ?

Mit der These vom Übergang von der fordistischen Massen- zur differenzierten Qualitätsproduktion wird versucht, die Reaktionen der Unternehmen auf diese Veränderungen in der Gesellschaft und auf den Märkten zu beschreiben, anknüpfend zum Beispiel an Überlegungen der Regulationstheorie. In nachfragedominierten, gesättigten Märkten steigt die Macht der Kunden, nicht nur der Endkunden, sondern auch der System-Hersteller gegenüber ihren Zulieferern. Im Zuge der Ausdifferenzierung der Gesellschaft
(Individualisierung) werden die Märkte unübersichtlicher, segmentierter und dynamischer. Einige Elemente dieser natürlich als idealtypisch zu verstehenden Unterscheidungen sind in Tabelle 1 wiedergegeben. Die Verankerung des Nachhaltigkeitsziels in der strategischen Unternehmensplanung dürfte umso notwendiger und erfolgreicher sein, je mehr Aspekte aus der dritten Spalte für die betreffenden Märkte bzw. für die betreffende Unternehmensstrategie zutreffen.

Selbstverständlich bietet diese Typisierung nur ein Suchraster. In der Unternehmensrealität lassen sich Qualität und Kosten nicht trennen. Unternehmen haben immer mit beiden Anforderungen gleichzeitig zu kämpfen. Es geht einerseits um Kostenvorteile und Kostenreduktion durch Rationalisierung und Standardisierung (economies of scale), und zugleich darum, mit einer breiten Produkt(varianten)palette der Nachfrage entgegen zu kommen (economies of scope).

Eine auch mit Blick auf Nachhaltigkeit hochinteressante Möglichkeit stellt angesichts dieser Zwickmühle die Plattformstrategie dar. Auf einer in Massenproduktion hergestellten Plattform werden erst im zweiten Produktionsabschnitt all die vielen nötigen Varianten realisiert. Eine wichtige Voraussetzung dafür ist wiederum die Modulbauweise, die - wieder mit Blick auf Nachhaltigkeit - die Spielräume für lange Nutzungszeiten, für Reparaturen und vor allem Modernisierungen eröffnet. Einer unserer Kooperationspartner,

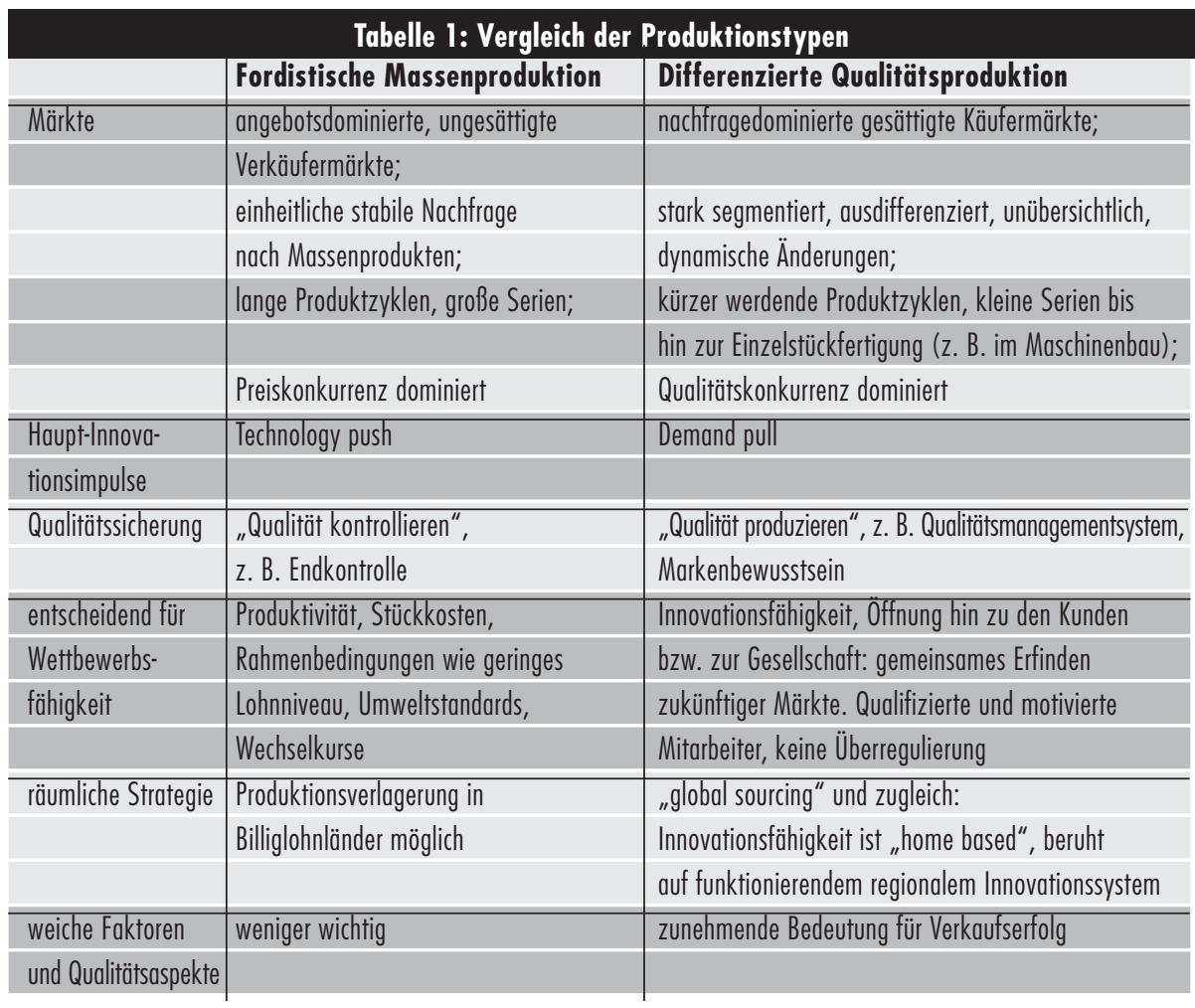


die Firma Jungheinrich, könnte damit zum Beispiel Gabelstapler auf den Markt bringen, in die wahlweise ein Diesel-, Gas-, Elektro- oder Brennstoffzellenantrieb integriert werden kann, mit Nachrüstbarkeitsgarantie!

\section{Gewinner und Verlierer}

Natürlich hat das Anknüpfen an der Wettbewerbsfähigkeit im Rahmen von Nachhaltigkeitsstrategien auch seine Kehrseiten. Die Palette möglicher Ansprechpartner für Nachhaltigkeitsstrategien bleibt auch hier eingeschränkt, auf ,,moderne“ Unternehmen, die sich erfolgreich in nachfragedominierten Märkten bewegen (wollen). Jedoch ist weder die langfristige Strategie- noch die Innovationsfähigkeit ein Privileg von Großunternehmen. Viel wesentlicher ist die interne Struktur, die Qualität des Managements und der Mitarbeiter und die Kommunikationsfähigkeit mit der Unternehmensumwelt.

Das größere Problem, den Herausforderungen der Nachhaltigkeit mit Wettbewerbsstrategien zu begegnen, liegt allerdings darin, dass es im Wettbewerb nicht nur Gewinner geben kann. Es wird auch Verlierer geben. Diese Verlierer werden Unternehmen mit ihren Beschäftigten und deren Familien sein, und zwar sowohl hier in den Industrienationen als auch in den Entwicklungs- und Schwellenländern. Die weitere quantitative und qualitative Intensivierung des Wettbewerbs wird ganze Volkswirtschaften treffen.

Eine umfassende Nachhaltigkeitsstrategie ist allein mit Unternehmenskooperationen und dem Anknüpfungspunkt Wettbewerbsfähigkeit also nicht zu realisieren. Sie ist darauf angewiesen, dass der Wettbewerb eingebunden wird in einen Rahmen, der soziale und ökologische Mindeststandards sichert. Staatliche und zwischenstaatliche Regulation, die Verbraucher mit ihrer Verbrauchermacht und nicht zuletzt die Nicht-Regierungs-Organisationen sind wichtige Akteure bei dieser Rahmensetzung (2). Die Demonstranten in Seattle haben auf die Notwendigkeit einer internationalen Handelsordnung unter Einbeziehung aller normativen Elemente des Nachhaltigkeitsziels noch einmal deutlich hingewiesen.

\section{Anmerkungen}

(1) Das Projekt wird vom Bundesforschungsministerium im Programm Modellprojekte Nachhaltigen Wirtschaftens geför- dert. Der Zwischenbericht ist im Internet erhältlich unter http://www.nachhaltige-metallwirtschaft.de

(2) Vgl. von Gleich, Arnim: Zwischen Überleben und Gutem Leben. Leitbilder und Strategien der Nachhaltigkeit. In: Ökologisches Wirtschaften 3-4/2000, S. 25-26.

\section{Die Autoren}

Dr. Arnim von Gleich ist Professor für Technikbewertung an der Fachhochschule Hamburg.

Kontakt: FH Hamburg, FB Maschinenbau und Produktion, Berliner Tor 21, 20099 Hamburg, Tel. 040/ 42859-4345, Fax -2658, E-mail: gleich@rzbt.fhhamburg.de

Manuel Gottschick ist wissenschaftlicher Mitarbeiter der Universität Hamburg im FB Informatik, Arbeitsbereich Angewandte und Sozialorientierte Informatik. E-mail: gottschick@informatik.uni-hamburg.de Dirk Jepsen ist Mitarbeiter des Ökopol-Instituts Hamburg. E-mail: d-jepsen@oekopol.de

\section{Selbstbewertung mit Anspruch}

\section{Wie kann die Umweltleistung eines Unternehmens so gemessen werden, dass tatsächlich Fortschritte auf dem Weg zur Nachhaltigkeit damit abgebildet wer- den können? Das hierzu vorgestellte Instrument beruht auf einer Selbstbewer- tung durch die Unternehmen und zeichnet sich durch drei Besonderheiten aus: Es hebt strategische und produktbezogene Aspekte hervor, berücksichtigt neben der Öko-Effizienz auch die Öko-Effektivität und kombiniert reale und monetäre Größen. Interessante Perspektiven verspricht eine Kombination mit dem Kon- zept der Balanced Scorecard.}

$\mathrm{V}$ Von Jens Clausen und Volker Stablmann on Umweltleistung zu sprechen ist problematisch. Menschen und von Menschen geschaffene Organisationen wie Unternehmen nehmen zuallererst und ständig Leistungen der Natur in Anspruch. Diese werden wiederum umgewandelt zu wirklichen oder vermeintlichen Nutzenstiftungen für den Menschen. Sieht man die ursprüngliche Wertschöpfungsgrundlage in der „natürlichen Natur“ (Günter Altner) und nicht in Unternehmen, dann heißt Umweltleistung, dass der Naturkapitalstock erhalten bleibt oder sogar gestärkt wird, dass die vorhandenen Umweltbelastungen absolut reduziert werden und ein möglichst geschlossener Stoffkreislauf mit der Natur entsteht. Ökonomie ist nach diesem Verständnis nicht nur monetär, sondern physisch, ökologisch, soziologisch und philosophisch erklärungsbedürftig. Sie ist langfristig angelegt und nicht allein erwerbswirtschaftlich, sondern auch selbstversorgend, nicht beherrschend, sondern eingeordnet in kulturelle Vielfalt und verbunden mit einem nachhaltigen Lebensstil. Mit anderen Worten lässt sich ein Urteil über die Umweltlei- stung nicht abgeben, ohne die Klärung der Wertgrundlagen unterschiedlicher ökonomieverständnisse. Hierfür ist insbesondere die Gegenüberstellung des herrschenden Mainstreams Neoklassik/ Neoliberalismus auf der einen Seite mit der aufkeimenden ökologischen Ökonomik auf der anderen Seite von Bedeutung.

Auf dieser Ebene hinterfragt, gerät die Konzeption einer Methodik zur Messung der Umweltleistung nicht einfach. In Kooperation mit fünf mittelständischen Öko-Pionier-Unternehmen und gefördert durch die Deutsche Bundesstiftung Umwelt haben wir uns an die komplexe Aufgabe gewagt (1).

\section{Die Systematik der Messung}

Zur ersten Operationalisierung des Oberziels nachhaltige Entwicklung wurden die Konturen eines nachhaltigen Unternehmens entworfen. Um eine kontinuierliche Selbstbewertung zu ermöglichen, haben wir das Managementmodell der European Foundation for Quality Management zugrunde gelegt (2), das aber sowohl im Bereich der Befähiger als auch der Ergebnisse entscheidend ergänzt und vertieft wurde (siehe Abb. 1 auf S. 20).

Die entwickelte Methodik zur Umweltleistungsmessung besteht aus einem branchenübergreifenden Kriterienkatalog mit 142 Fragen zur Beur- 
(c) 20I0 Authors; licensee IÖW and oekom verlag. This is an article distributed under the terms of the Creative Commons Attribution Non-Commercial No Derivates License (http://creativecommons.org/licenses/by-nc-nd/3.o/), which permits unrestricted use, distribution, and reproduction in any medium, provided the original work is properly cited. 